Supporting Information

\title{
Kinetically Controlled Synthesis of Triangular and Hexagonal Nanoplates of Pd and Their SPR/SERS Properties
}

\author{
Yujie Xiong, ${ }^{\dagger}$ Joseph M. McLellan, ${ }^{\dagger}$ Jingyi Chen, ${ }^{\dagger}$ Yadong Yin, ${ }^{\dagger}$ \\ Zhi-Yuan $\mathrm{Li}^{\S}{ }^{\S}$ and Younan $\mathrm{Xia}^{\dagger}{ }^{\dagger} *$ \\ ${ }^{\dagger}$ Department of Chemistry, University of Washington, Seattle, Washington 98195 \\ The Molecular Foundry, Lawrence Berkeley National Laboratory, Berkeley, California 94720 \\ ${ }^{\S}$ Institute of Physics, Chinese Academy of Sciences, Beijing 100080, P. R. China \\ *Corresponding author. E-mail: xia@chem.washington.edu
}




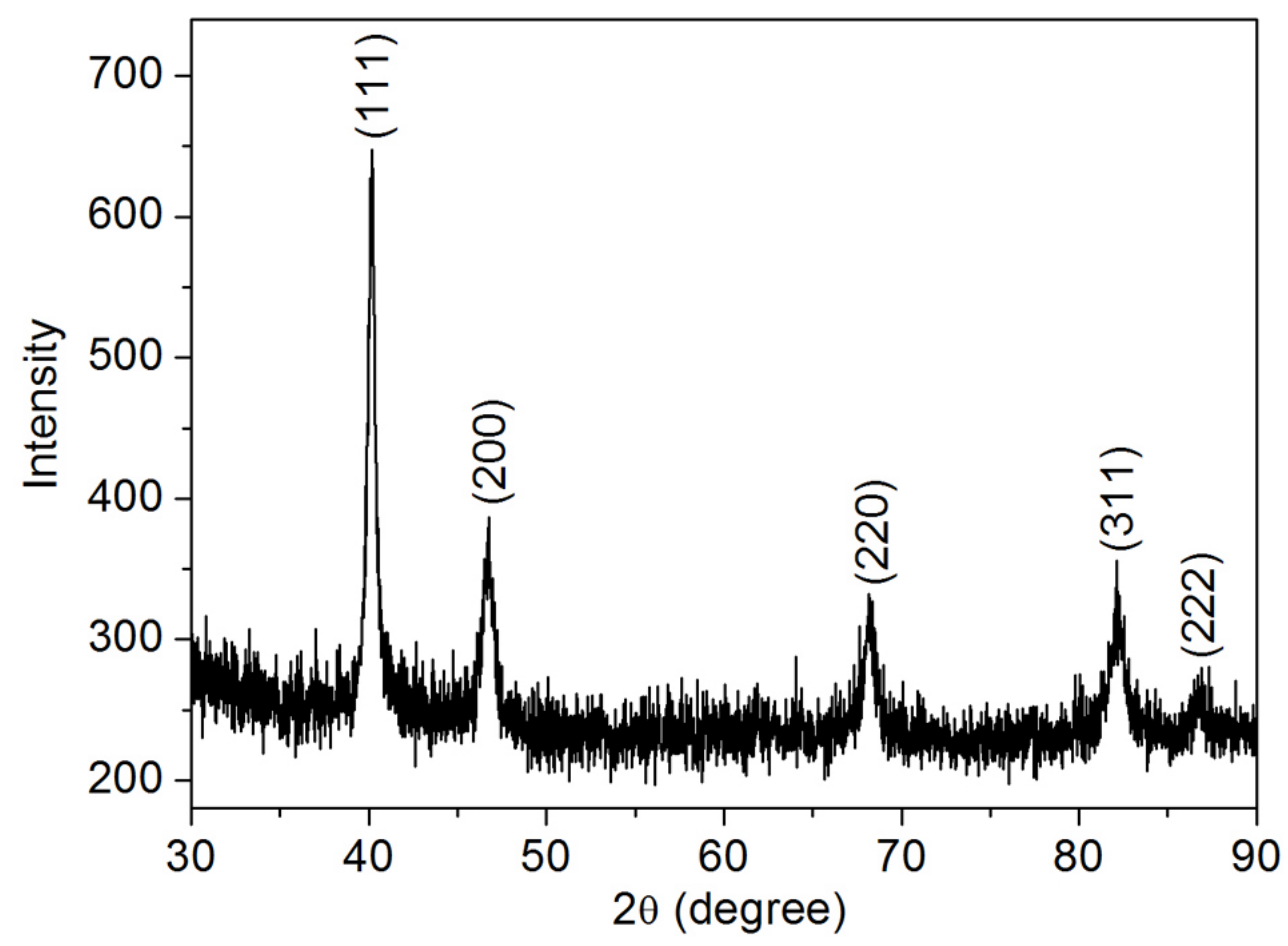

Figure S1. Powder XRD pattern of the as-prepared triangular nanoplates. All the peaks can be indexed to $f c c$ Pd (JCPDS card, 05-0681). The ratio between the intensities of (111) and (200) peaks is much higher than the index value (2.89 versus 2.38 ), indicating that the top and bottom faces of each nanoplate are bounded by $\{111\}$ planes. These nanoplates are more or less oriented parallel to the supporting substrate, resulting in a stronger (111) diffraction peak than that of a conventional powder sample. 

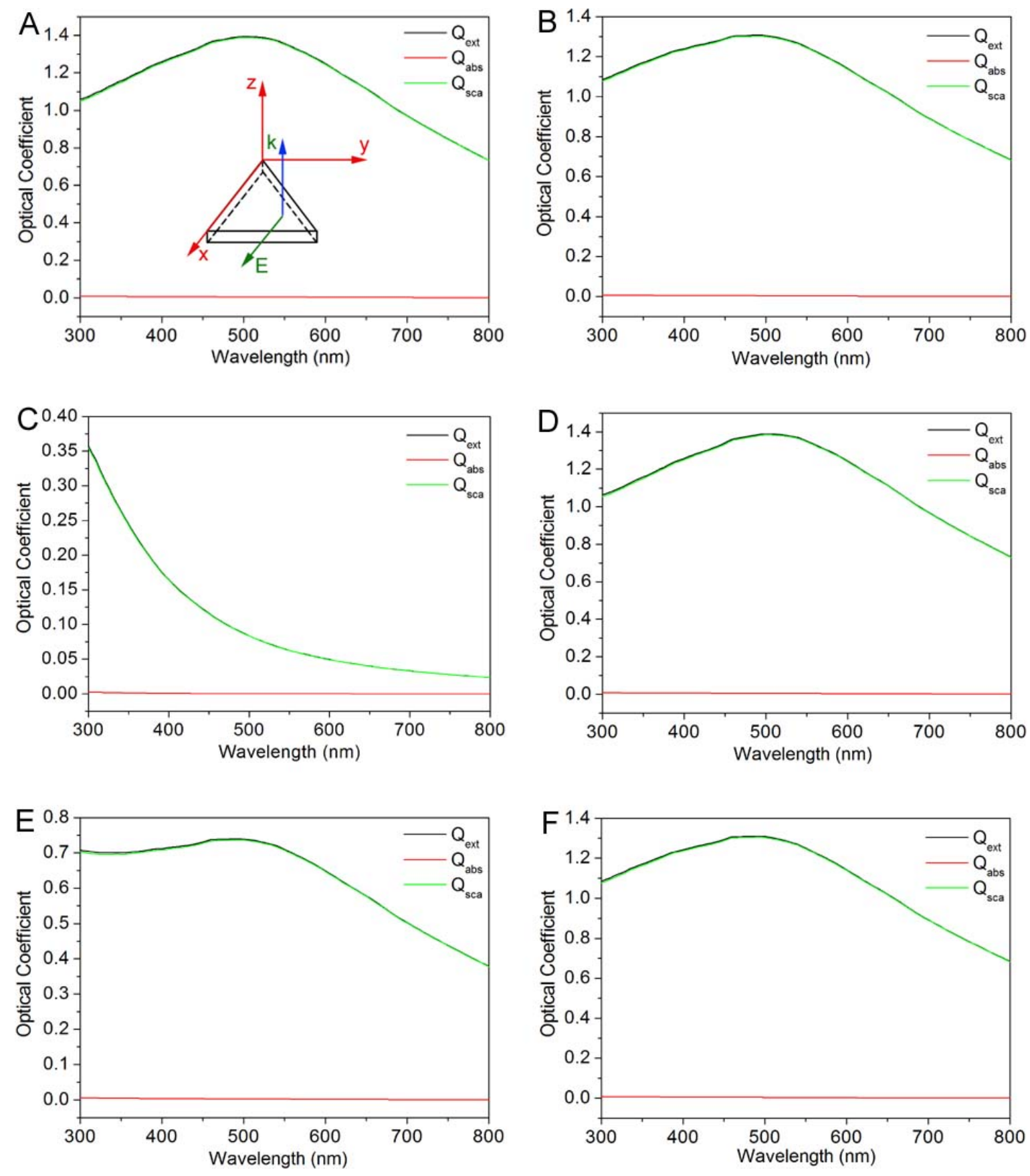

Figure S2. The extinction, absorption, and scattering coefficients of a triangular nanoplate as calculated using the DDA method with six different configurations. In the calculations, we assume that the triangular plate is normal to the z-axis and one of its edges is parallel to the $\mathrm{x}$ axis as depicted in the inset of panel $\mathrm{A}$. The configurations are: (A) incident light along $\mathrm{k}=$ $(0,0,1)$ and electric field polarization $\mathrm{E}=(1,0,0) ;(\mathrm{B}) \mathrm{k}=(0,0,1)$ and $\mathrm{E}=(0,1,0) ;(\mathrm{C}) \mathrm{k}=(0,1,0)$ and $\mathrm{E}=(0,1,0)$; (D) $\mathrm{k}=(0,1,0)$ and $\mathrm{E}=(1,0,0)$; $(\mathrm{E}) \mathrm{k}=(1,0,1)$ and $\mathrm{E}=(-1,0,1)$; and $(\mathrm{F}) \mathrm{k}=$ $(1,0,1)$ and $\mathrm{E}=(0,1,0)$. The optical coefficients were defined as $\mathrm{C} / \pi \alpha_{\mathrm{eff}}{ }^{2}$ (with $\mathrm{C}$ being crosssections obtained directly from DDA calculation, and with $\alpha_{\text {eff }}$ being defined through the concept of an effective volume equal to $4 \pi \alpha_{\mathrm{eff}}{ }^{3} / 3$ for the plate). 